\title{
Cerebral Palsy
}

National Institute of Neurological Disorders and Stroke (NINDS)

\section{Source}

National Institute of Neurological Disorders and Stroke (NINDS). Cerebral Palsy

Information Page.

The term cerebral palsy refers to a group of neurological disorders that appear in infancy or early childhood and permanently affect body movement, muscle coordination, and balance. CP affects the part of the brain that controls muscle movements. The majority of children with cerebral palsy are born with it, although it may not be detected until months or years later. The early signs of cerebral palsy usually appear before a child reaches 3 years of age. The most common are a lack of muscle coordination when performing voluntary movements (ataxia); stiff or tight muscles and exaggerated reflexes (spasticity); walking with one foot or leg dragging; walking on the toes, a crouched gait, or a "scissored" gait; and muscle tone that is either too stiff or too floppy. Other neurological symptoms that commonly occur in individuals with CP include seizures, hearing loss and impaired vision, bladder and bowel control issues, and pain and abnormal sensations. A small number of children have $\mathrm{CP}$ as the result of brain damage in the first few months or years of life, brain infections such as bacterial meningitis or viral encephalitis, or head injury from a motor vehicle accident, a fall, or child abuse. The disorder isn't progressive, meaning that the brain damage typically doesn't get worse over time. Risk factors associated with CP do not cause the disorder but can increase a child's chance of being born with the disorder.CP is not hereditary. 